\title{
Análisis en estudios de cohorte: ¿usar odds ratio o riesgo relativo?
}

\section{Analyses in cohort studies: to use odds ratios or relative risks?}

Correspondencia Joshua Peñafiel-Sam U201211120@upc.edu.pe

Recibido: 19/12/2016 Aprobado: 21/12/2016

Citar como: Chang-Cabanillas S, Peñafiel-Sam J, AlarcónGuevara S. Análisis en estudios de cohorte: iusar odds ratio 0 riesgo relativo? Acta Med Peru. 2016;33(4):336-7
Sergio Chang-Cabanillas ${ }^{1}$, Joshua Peñafiel-Sam¹, Samuel Alarcón-Guevara1

1 Escuela de Medicina, Universidad Peruana de Ciencias Aplicadas. Lima, Perú.

\section{Sr. Editor:}

El artículo publicado por Granda y cols. ${ }^{[1]}$ presenta un análisis retrospectivo de casos de hernia inguinal tratados quirúrgicamente con una de dos técnicas en el Hospital Militar Central de Lima, Perú. El estudio mencionado, en nuestra opinión, posee varios puntos merecedores de comentarios especialmente sobre el análisis estadístico realizado.

A pesar de ser presentado como un censo de los pacientes tratados durante el periodo 20122014, recalcamos la necesidad de estimar siempre un tamaño de muestra y/o un cálculo de poder estadístico. En primer lugar, para obtener el número mínimo de personas necesarias a incluir para detectar tanto el desenlace (complicaciones) como alguna asociación específica (por ejemplo: recurrencia con tamaño de la hernia) en magnitudes pre-establecidas ${ }^{[2]}$. En segundo lugar, para entender mejor la capacidad de la muestra de detectar alguna asociación si es que esta existe.

De igual modo, no se tomaron en cuenta variables importantes como el riesgo quirúrgico Goldman y la clasificación ASA (American Society of Anesthesiologists), que podría explicar la mayor o menor incidencia de complicaciones como se ha reportado en otro estudio similar por Adelsdorfer y cols. [3]. Asumimos que estos datos regularmente están disponibles en las historias clínicas al ser parte de la evaluación rutinaria de los pacientes quirúrgicos en general y podrían ser incorporados en un eventual reanálisis de los datos.

Finalmente, y sobre lo cual se centra nuestra principal crítica, en el análisis de asociación se calcularon los odds ratio (OR), lo cual no es necesariamente la mejor opción. El problema es que, cuando la frecuencia del desenlace es alta, este estimador tiende a mostrar resultados alejados de la hipótesis nula (estimador igual a uno) en comparación a lo que se obtiene al calcular una razón de riesgos o incidencias (RR) en estudios de cohorte ${ }^{[4]}$. En el caso del estudio de Granda y cols., la frecuencia es de $15,8 \%$ a $17,8 \%$ para las complicaciones tardías agregadas, y de $12,4 \%$ a $13,9 \%$ para la neuralgia específicamente, dependiendo de la población analizada según su Tabla 
3. Esto nos sugiere que, en un eventual recálculo de los RR, es muy probable que muchas de estas asociaciones disminuyan en su magnitud. Este recálculo convendría para interpretar más francamente la importancia clínica de los hallazgos. Aunque existen métodos para aproximar un recálculo del RR a partir de un OR reportado [5], lamentablemente no pudimos realizarlo puesto que no toda la información requerida para este efecto fue reportada en el artículo de Granda y cols.

En síntesis, desde nuestra apreciación, este estudio presenta serias limitaciones en su abordaje epidemiológicocuantitativo y sus resultados no necesariamente reflejan el verdadero fenómeno bajo estudio. A pesar de esto, el estudio aporta a la literatura local, pero hay que tener en cuenta lo discutido en la presente carta. Futuros estudios sobre el tema deberían tomar en cuenta los aspectos mencionados.

\section{REFERENCIAS BIBLIOGRÁFICAS}

1. Granda AC, Correa-Tineo S, Quispe AM. Herniorrafía con la técnica de Lichtenstein versus Nyhus en el manejo de las hernias inguinales y sus complicaciones postoperatorias en un hospital del Perú. Acta Med Peru. 2016;33(3):208-16.

2. Guerra-Márquez A, Carrillo-Montes MG. Conceptos básicos y premisas para calcular el tamaño de muestra. Rev Med Inst Mex Seguro Soc. 2006;44(2):67-9.

3. Adelsdorfer CO, Slako MM, Klinger JR, Carter JM, Bergh CO, Benavides CJ. Complicaciones postoperatorias de la serie prospectiva de pacientes con hernioplastia inguinal, en protocolo de hospitalización acortada del Hospital Dr. Gustavo Fricke de Viña del Mar. Rev Chil Cir. 2007;59(6):436-42.

4. Cummings P. The relative merits of risk ratios and odds ratios. Arch Pediatr Adolesc Med. 2009;163(5):438-45.

5. Zhang J, Yu KF. What's the relative risk? A method of correcting the odds ratio in cohort studies of common outcomes. JAMA. 1998;280(19):1690-1.

\section{Ahora puede enviar sus artículos para Acta Médica Peruana en nuestro Open Journal System:}

www.amp.cmp.org.pe

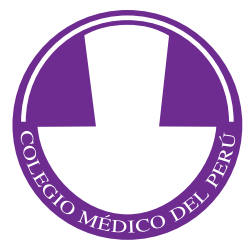

